\title{
Clinical outcomes after percutaneous coronary intervention for early versus late and very late stent thrombosis: a systematic review and meta-analysis
}

\author{
Yi-Xing Yang ${ }^{1} \cdot$ Yin Liu $^{2} \cdot$ Xiao-Wei $\mathrm{Li}^{2} \cdot$ Peng-Ju Lu² $\cdot$ Jiao Wang ${ }^{1} \cdot$ Chang-Ping $\mathrm{Li}^{1} \cdot$ Jing Gao
}

Published online: 20 July 2020

(c) The Author(s) 2020

\begin{abstract}
Whether the clinical outcomes of stent thrombosis (ST) are different when stratified by time of occurrence remains unclear. The objective of this study was to compare the short- and long-term clinical outcomes after percutaneous coronary intervention (PCI) for early stent thrombosis (EST) versus late stent thrombosis (LST) and very late stent thrombosis (VLST). We enrolled eligible studies searched from the main electronic databases (EMBASE, PubMed, Cochrane). The primary endpoints were in-hospital, 30-day, 1-year and long-term mortality. The secondary endpoints included recurrent stent thrombosis (RST) and target vessel/lesion revascularization (TVR/TLR) during hospitalization, at 30 days, at 1 year and at long-term follow-up. A total of 23 studies with 17,592 patients were included. Compared with mortality rates of the late and very late thrombosis (LST/VLST) group, in-hospital $(\mathrm{P}=0.004)$, 30-day $(\mathrm{P}<0.00001), 1$-year $(\mathrm{P}<0.00001)$ and long-term mortality rates $(\mathrm{P}=0.04)$ were significantly higher in the EST group. The in-hospital TVR/TLR rates were similar between the EST group and the LST/VLST group. However, a higher trend in TVR/TLR rate at 30 days and a significantly higher TVR/TLR rate at 1 year $(\mathrm{P}=0.002)$ as well as at long-term follow up $(\mathrm{P}=0.009)$ were found in the EST group. EST patients also trended toward higher risk of RST in both short- and long-term follow-up than LST/VLST patients, although differences were not statistically significant. After PCI treatment, patients with EST have worse clinical outcomes in both short- and long-term follow-up than patients with LST/VLST. Further studies are warranted to determine the optimal treatment strategies for EST.
\end{abstract}

Keywords Stent thrombosis · Outcomes · Percutaneous coronary intervention · Meta-analysis

Yi-Xing Yang and Yin Liu have contributed equally to this work

Electronic supplementary material The online version of this article (https://doi.org/10.1007/s11239-020-02184-7) contains supplementary material, which is available to authorized users.

PROSPERO registration number CRD42019144994.

Chang-Ping Li

lichangping@tmu.edu.cn

$\triangle$ Jing Gao gaojing2088@163.com

1 Tianjin Medical University, No.22 Qi xiang tai Road, Heping District, Tianjin 300070, People's Republic of China

2 Department of Cardiology, Tianjin Chest Hospital, No. 261 Tai er zhuang Road, Jinnan District, Tianjin 300222, People's Republic of China

3 Cardiovascular Institute, Tianjin Chest Hospital, No.261 Tai er zhuang Road, Jinnan District, Tianjin 300222, People's Republic of China

\section{Highlights}

- This is the first meta-analysis to investigate the associations between the timing of ST occurrence and the clinical outcomes of ST.

- Patients with EST have worse clinical outcomes in both short- and long-term follow-up than patients with LST/ VLST.

- Further studies are warranted to determine the optimal treatment strategies for EST.

\section{Introduction}

Stent thrombosis (ST) is a rare but catastrophic complication of PCI with high mortality in both short-term and long-term periods [1, 2]. According to the Academic Research Consortium criteria, ST can be stratified into early stent thrombosis 
(EST), occurring within 30 days after index PCI, late stent thrombosis (LST), occurring from 30 days to 1 year after index PCI, and very late stent thrombosis (VLST), occurring more than 1 year after index PCI [3]. Recently, several studies investigated the associations between the timing of ST occurrence and the clinical outcomes of ST, but the results were inconsistent [4-26]. Therefore, we conducted a meta-analysis to compare the short-term and long-term clinical outcomes following PCI for patients with EST versus patients with LST and VLST.

\section{Methods}

A study protocol was developed prior to data collection and was registered on PROSPERO and can be accessed at https://www.crd.york.ac.uk/prospero/display_recor d.php?ID=CRD42019144994.

\section{Search strategy and study selection}

We searched the literature in the PUBMED, EMBASE and Cochrane Library databases, using combinations of the following key words: "outcome" OR "prognosis" AND "early stent thrombosis" OR "acute stent thrombosis" OR "subacute stent thrombosis" OR "late stent thrombosis." An initial screen of titles and abstracts was conducted to exclude studies that were irrelevant to the present study. Full-text of the relevant articles were evaluated by the selection criteria. Studies were eligible for inclusion if they: (1) compared the clinical outcomes of EST versus LST or VLST; (2) had angiographically confirmed (definitive) ST; (3) included PCI treatment for ST; (4) follow-up time including in-hospital, 30-day, 1-year and long-term periods ( $>1$ year); (5) had at least 30 participants; and (6) were randomized clinical trials, observational studies or abstracts with sufficient data. Studies were excluded if they: (1) did not compare the clinical outcomes of EST versus LST or VLST; (2) included probable or possible ST; (3) included unclear treatment for ST; (4) had other follow-up periods such as 7-day, 180-day, etc.; (5) participants were fewer than 30; and (6) were categorized as case reports or comments. In addition, reference lists of the selected studies were also screened for potential articles.

\section{Data extraction and quality assessment}

Data extraction was performed using a standardized data collection form. The primary endpoints were in-hospital, 30-day, 1-year and long-term mortality. The secondary endpoints included RST and TVR/TLR during hospitalization, at 30 days, at 1 year and at long-term follow-up. Definitions of "ST", "RST", "TVR" and "TLR" corresponded with the Academic Research Consortium criteria [3]. Study quality was assessed by using the Newcastle-Ottawa Scale [27]. Two reviewers independently performed the study search and selection, data extraction and quality assessment of the selected studies. Disagreements were resolved by team discussion.

\section{Statistical analysis}

Results were analyzed using computed pooled risk ratios (RR) with 95\% confidence intervals (CIs). Statistical heterogeneity was evaluated by the Cochrane $\mathrm{Q}$ test and the $\mathrm{I}^{2}$ statistic. A random-effect model was used when a significant heterogeneity $\left(\mathrm{P}<0.05\right.$ or $\left.\mathrm{I}^{2}>50\right)$ was detected, otherwise, a fixed-effect model was used ( $\mathrm{P} \geq 0.05$ or $\left.\mathrm{I}^{2} \leq 50 \%\right)$. To analyze intuitively, LST and VLST patients were combined as the control group for EST. Statistical analysis was carried out using the REVIEW MANAGER software (Version 5.3, Cochrane Collaborative, Oxford, England).

\section{Results}

\section{Study characteristics}

The literature search strategy process is shown in Fig. 1. From the 4306 published studies identified, 21 observational studies and 2 abstracts with a total of 17,592 patients were finally enrolled in our analyses. Among the patients enrolled,

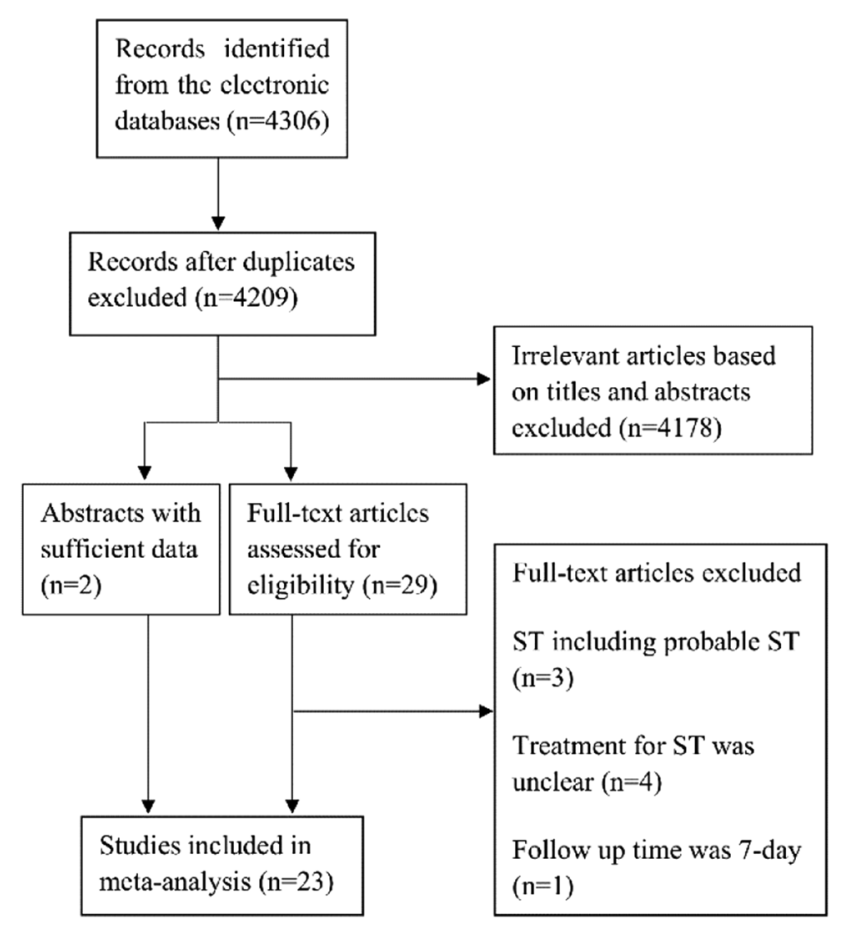

Fig. 1 Flow diagram of literature search strategy process 
4937 patients had EST, and 12,655 patients had LST/VLST. The main characteristics of the included studies are shown in Table 1. Quality assessment of the studies is shown in Supplementary Table 1.

\section{Patients' characteristics}

Table 2 shows the baseline clinical characteristics of patients in the two groups. Compared with those with LST/VLST, patients with EST were more frequently diabetics and presented with cardiogenic shock (CS) at the time of ST (diabetics: $41.6 \%$ vs. $31.3 \%, \mathrm{P}=0.0004$, 13 studies including 15,905 patients were used for this analysis, Supplemental Fig. 1a); (CS: $13.7 \%$ vs. 8.9\%,
$\mathrm{P}<0.00001,10$ studies including 14,181 patients contributed to this analysis, Supplemental Fig. 1b). However, male gender and hyperlipemia were more frequent in patients with LST/VLST than in those with EST (male: $77.0 \%$ vs. $72.1 \%, \mathrm{P}=0.03,13$ studies including 15,907 patients were used for this analysis, Supplemental Fig. 2a); (hyperlipemia: $85.7 \%$ vs. $71.4 \%, \mathrm{P}<0.00001,9$ studies including 9217 patients contributed to this analysis, Supplemental Fig. 2b). Four studies including 8188 patients reported that chronic kidney disease (CKD) was higher in the EST group than in the LST/VLST group (4.6\% vs. $2.3 \%, \mathrm{P}<0.00001$, Supplemental Fig. 3a), while another seven studies including 2088 patients reported the incidence of CKD was markedly higher in the LST/VLST

Table 1 Main characteristics of the included studies

\begin{tabular}{|c|c|c|c|c|c|c|c|c|}
\hline Author & Year & Study type & $\mathrm{PN}$ & Initial stent type & Type of ST & Manifestation of ST & $\begin{array}{l}\text { Treatment } \\
\text { for ST }\end{array}$ & $\begin{array}{l}\text { Follow up time and end- } \\
\text { points }\end{array}$ \\
\hline Lemesle [4] & 2009 & Observational & 91 & DES & Definite & STEMI $74.7 \%$ & PCI & $\begin{array}{l}\text { IH/1 M/1Y death, RST, MI, } \\
\text { MACE }\end{array}$ \\
\hline Margolis [5] & 2016 & Observational & 83 & NA & Definite & STEMI $100 \%$ & PCI & $1 \mathrm{M}$ death \\
\hline Jones [6] & 2013 & Observational & 194 & DES/BMS & definite & STEMI $100 \%$ & PCI & LT MACE \\
\hline Kimura [7] & 2010 & Observational & 611 & DES & Definite & STEMI $69.0 \%$ & PCI & $1 \mathrm{M} / 1 \mathrm{Y} / \mathrm{LT}$ death \\
\hline Kubo [8] & 2014 & Observational & 152 & DES/BMS & Definite & AMI $81.6 \%$ & PCI & $\begin{array}{l}\text { IH/1Y/LT death; 1Y/LT } \\
\text { TLR, CD, MACE; LT } \\
\text { RST }\end{array}$ \\
\hline Armstrong [9] & 2012 & Observational & 7079 & DES/BMS & Definite & $\begin{array}{l}\text { STEMI } 64.2 \% \\
\text { (AMI } 87.1 \% \text { ) }\end{array}$ & PCI & IH death \\
\hline de la TH [10] & 2008 & Observational & 301 & DES & Definite & STEMI $83.7 \%$ & PCI & IH/LT death, RST \\
\hline Daemen [11] & 2007 & Observational & 152 & DES & Definite & AMI $45.4 \%$ & PCI & IH/1 M death, RST, TVR \\
\hline Singh [12] & 2018 & Observational & 46 & DES/BMS & Definite & STEMI $82.6 \%$ & PCI & IH/LT death \\
\hline Kuramitsu [13] & 2019 & Observational & 313 & DES & Definite & NA & PCI & $1 \mathrm{M} / 1 \mathrm{Y} / \mathrm{LT}$ death, RST \\
\hline Mahmoud [14] & 2011 & Observational & 113 & DES/BMS & Definite & $\begin{array}{l}\text { STEMI } 85.0 \% \\
\text { (AMI } 100 \% \text { ) }\end{array}$ & PCI & $1 \mathrm{M} / 1 \mathrm{Y}$ death \\
\hline Lempereur [15] & 2016 & Observational & 101 & DES/BMS & Definite & STEMI $62.5 \%$ & PCI & $\begin{array}{l}1 \mathrm{M} / 1 \mathrm{Y} \text { death, TVR, } \\
\text { MACE }\end{array}$ \\
\hline $\operatorname{Kim}[16]$ & 2019 & Observational & 243 & DES/BMS & Definite & $\begin{array}{l}\text { STEMI } 63.8 \% \\
\text { (AMI } 89.7 \% \text { ) }\end{array}$ & PCI & 1Y MACE \\
\hline Armstrong [17] & 2014 & Observational & 656 & NA & Definite & NA & PCI & $1 \mathrm{M}$ death \\
\hline Almalla [18] & 2013 & Observational & 106 & DES/BMS & Definite & STEMI $78.3 \%$ & PCI & LT MACE \\
\hline Van Werkum [19] & 2009 & Observational & 431 & DES/BMS & Definite & NA & PCI & LT MACE \\
\hline Katsikis [20] & 2019 & Observational & 131 & DES/BMS & Definite & STEMI $88.0 \%$ & PCI & LT death \\
\hline Yeo [21] & 2015 & Observational & 210 & DES/BMS & Definite & $\begin{array}{l}\text { STEMI } 65.0 \% \\
\text { (AMI } 90.0 \%)\end{array}$ & PCI & LT MACE \\
\hline Konishi [22] & 2019 & Observational & 370 & DES & Definite & AMI $29.5 \%$ & PCI & IH death \\
\hline Tovar Forero [23] & 2019 & Observational & 679 & DES/BMS & Definite & AMI $87.2 \%$ & PCI & LT MACE \\
\hline Feldman [24] & 2011 & Abstract & 5319 & DES/BMS & Definite & $\begin{array}{l}\text { STEMI } 62.2 \% \\
\text { (AMI } 84.8 \% \text { ) }\end{array}$ & PCI & IH death \\
\hline Shimotakahara [25] & 2013 & Abstract & 102 & BMS & Definite & NA & PCI & LT death, TLR \\
\hline Kukreja [26] & 2009 & Observational & 109 & DES/BMS & Definite & NA & PCI & LT death \\
\hline
\end{tabular}

$P N$ patient number, $S T$ stent thrombosis, $D E S$ drug-eluting stent, $B M S$ bare-metal stent, $N A$ not available, $I H$ in-hospital, $P C I$ percutaneous coronary intervention, $1 M 1$ month, $I Y 1$ year, $L T$ long-term, MACE major adverse cardiovascular event, $R S T$ recurrent stent thrombosis, $A M I$ acute myocardial infarction, STEMI ST segment elevation myocardial infarction, TVR target vessel revascularization, TLR target lesion revascularization, $C D$ cardiac death, $M I$ myocardial infarction 
Table 2 Baseline clinical characteristics of patients

\begin{tabular}{|c|c|c|c|c|c|c|c|c|c|c|c|c|c|c|c|c|}
\hline \multirow[t]{2}{*}{ Study } & \multicolumn{2}{|l|}{$\mathrm{PN}$} & \multicolumn{2}{|c|}{ MA (years) } & \multicolumn{2}{|c|}{ Male $(\%)$} & \multicolumn{2}{|c|}{ HTN $(\%)$} & \multicolumn{2}{|c|}{ DM (\%) } & \multicolumn{2}{|c|}{$\operatorname{HLP}(\%)$} & \multicolumn{2}{|c|}{ CS $(\%)$} & \multicolumn{2}{|l|}{ CKD $(\%)$} \\
\hline & EST & LST & EST & LST & EST & LST & EST & LST & EST & LST & ETS & LST & EST & LST & EST & LST \\
\hline emesle [4] & 51 & 40 & 61.4 & 63.5 & 51.0 & 70.0 & 86.3 & 82.5 & 54.9 & 47.5 & 88.2 & 95.0 & 39.2 & 20 & 21.6 & 22.5 \\
\hline Marg & 35 & 48 & 66.9 & 65.2 & 83.0 & 87.0 & 71.0 & 73.0 & 37.0 & 31.0 & 71.0 & 87.0 & NA* & & NA* & \\
\hline Jones [6] & 67 & 127 & $62.6^{*}$ & & $71.0^{*}$ & & $57.5 *$ & & $26.0^{*}$ & & $55.0^{*}$ & & $6.2^{*}$ & & NA* & \\
\hline Kimura [7] & 322 & 289 & 67.1 & 64.8 & 81.0 & 83.0 & 72.0 & 75.0 & 43.0 & 40.0 & $\mathrm{NA}^{*}$ & & 11.0 & 5.9 & 6.8 & 19 \\
\hline Kubo [8] & 55 & 97 & 65.9 & 67.2 & 80.0 & 85.6 & 74.6 & 62.9 & 49.1 & 37.1 & 50.9 & 57.7 & 12.7 & 4.1 & 9.1 & 25.8 \\
\hline Armstrong [9] & 1391 & 5688 & 61.0 & 60.5 & 67.1 & 75.7 & 85.8 & 85.2 & 43.7 & 30.2 & 85.6 & 89.1 & 13.4 & 9.6 & 3.6 & 2.0 \\
\hline de la TH [10] & 149 & 152 & 62.5 & 58.4 & 60.0 & 78.3 & 53.0 & 45.4 & 40.0 & 21.7 & 43.0 & 53.3 & NA* & & 7.4 & 2.6 \\
\hline Daemen [11] & 91 & 61 & 61.9 & 58.0 & 73.0 & 80.0 & 39.0 & 46.0 & 28.0 & 8.0 & 45.0 & 54.0 & 9.0 & 8.0 & 9.0 & 2.0 \\
\hline Singh [12] & 38 & 8 & $58.6^{*}$ & & $73.9 *$ & & $45.7 *$ & & $45.7^{*}$ & & $32.6^{*}$ & & 18.4 & 12.5 & NA* & \\
\hline Kuramitsu [13] & 179 & 134 & 68.2 & 67.9 & 84.4 & 74.6 & 78.8 & 79.9 & 45.8 & 50.7 & 81.6 & 82.8 & 8.9 & 2.7 & 3.9 & 6.0 \\
\hline Mahmoud [14] & 59 & 54 & $63.5^{*}$ & & $77.0^{*}$ & & $44.2 *$ & & $13.3^{*}$ & & $43.4 *$ & & $\mathrm{NA}^{*}$ & & NA* & \\
\hline Lempereur [15] & 36 & 65 & 64.4 & 64.0 & 72.2 & 78.5 & 58.3 & 66.2 & 44.4 & 32.3 & NA* & & 19.4 & 9.2 & 0.0 & 1.5 \\
\hline Kim [16] & 110 & 133 & $64.4^{*}$ & & $69.5^{*}$ & & $59.7 *$ & & $40.7 *$ & & $9.1 *$ & & NA* & & $38.7 *$ & \\
\hline Armstrong [17] & 129 & 527 & 65.6 & 64.5 & 98.4 & 99.4 & $\mathrm{NA}^{*}$ & & 53.5 & 50.7 & NA* & & $\mathrm{NA}^{*}$ & & 9.3 & 4.7 \\
\hline Almalla [18] & 86 & 20 & $69.7 *$ & & $80.2 *$ & & $71.6^{*}$ & & $30.2 *$ & & NA* & & $28.3^{*}$ & & $20.8^{*}$ & \\
\hline Van Werkum [19] & 317 & 114 & $61.1^{*}$ & & $74.9 *$ & & $46.9^{*}$ & & $23.2 *$ & & $53.1 *$ & & $\mathrm{NA}^{*}$ & & $16.9^{*}$ & \\
\hline Katsikis [20] & 14 & 117 & $65.0 *$ & & $85.0 *$ & & $66.0^{*}$ & & $23.0 *$ & & $64.0^{*}$ & & $10.0^{*}$ & & $17.0^{*}$ & \\
\hline Yeo [21] & 69 & 141 & $61.0 *$ & & $86.0 *$ & & $76.0^{*}$ & & $39.0^{*}$ & & NA* & & $21.0^{*}$ & & $15.0^{*}$ & \\
\hline Konishi [22] & 287 & 83 & 68.5 & 69.2 & 80.4 & 81.3 & 51.1 & 65.1 & 40.2 & 41.0 & 41.2 & 48.2 & NA & & 2.7 & 22.9 \\
\hline Tovar Forero [23] & 345 & 334 & 64.1 & 61.4 & 74.2 & 79.9 & 51.2 & 54.8 & 23.6 & 20.7 & 54.1 & 66.2 & 16.9 & 6.9 & 17.8 & 21.5 \\
\hline Feldman [24] & 1012 & 4307 & 62.0 & 60.8 & 68.0 & 74.9 & $\mathrm{NA}^{*}$ & & 43.1 & 30.2 & NA* & & 13.5 & 8.6 & NA* & \\
\hline Shimotakahara [25] & 40 & 62 & $\mathrm{NA}^{*}$ & & $\mathrm{NA}^{*}$ & & $\mathrm{NA}^{*}$ & & $\mathrm{NA}^{*}$ & & $\mathrm{NA}^{*}$ & & NA* & & NA* & \\
\hline Kukreja [26] & 55 & 54 & $61.8 *$ & & $72.2 *$ & & $38.1 *$ & & $15.4^{*}$ & & $48.5^{*}$ & & NA* & & $\mathrm{NA}^{*}$ & \\
\hline
\end{tabular}

$P N$ patient number, $M A$ mean age, $H T N$ hypertension, $D M$ diabetes mellitus, $H L P$ hyperlipemia, $C S$ cardiogenic shock (at the time of ST), $C K D$ chronic kidney disease, EST early stent thrombosis, LST late stent thrombosis (including very late stent thrombosis here), NA not available

*Overall

group than in the EST group (21.5\% vs. $8.7 \%, \mathrm{P}=0.001$, Supplemental Fig. 3b).

Table 3 shows the lesion and treatment features of the two groups. Compared with the LST/VLST group, the EST group had a higher rate of bifurcation lesions and left anterior descending artery (LAD) lesions (bifurcation: 23.5\% vs. $15.2 \%, \mathrm{P}<0.00001,10$ studies including 10,272 patients were used for this analysis, Supplemental Fig. 4a); (LAD: $50.8 \%$ vs. $41.2 \%, \mathrm{P}<0.00001,11$ studies including 10,523 patients contributed to this analysis, Supplemental Fig. 4b). Additional stent (AS) was utilized more frequently in the LST/VLST group than in the EST group $(66.0 \%$ vs. $46.8 \%$, $\mathrm{P}<0.00001,13$ studies including 15,530 patients were used for this analysis, Supplemental Fig. 5a), whereas intra-aortic balloon pump (IABP) and glycoprotein IIb/IIIa inhibitor (GPI) were administered more frequently in the EST group than in the LST/VLST group (IABP: $17.3 \%$ vs. 9.5\%, $\mathrm{P}<0.0001,7$ studies including 9360 patients were used for this analysis, Supplemental Fig. 6a); (GPI: $70.2 \%$ vs. $65.5 \%, \mathrm{P}=0.02,6$ studies including 8333 patients contributed to this analysis, Supplemental Fig. 6b). No significant differences were found between the two groups in the rate of using thrombus aspiration (TA, LST/VLST $34.7 \%$ vs. EST $30.4 \%, \mathrm{P}=0.37,11$ studies including 14,945 patients were used for this analysis, Supplemental Fig. 5b). The rate of achieving thrombolysis in myocardial infarction (TIMI) grade 3 post-PCI was significantly lower in the EST group than in the LST/VLST group ( $88.3 \%$ vs. $92.6 \%, \mathrm{P}<0.00001$, 8 studies including 11,483 patients were used for this analysis, Supplemental Fig. 7).

\section{Primary endpoints}

Analysis of 8 studies including 13,510 patients demonstrated that in-hospital mortality was dramatically higher in the EST group than in the LST/VSLT group (RR: 1.67, 95\% CI 1.17-2.37, P=0.004, Fig. 2a). Analysis of 8 studies involving 2120 patients showed that 30-day mortality was significantly higher in the EST group than in the LST/ VLST group (RR: 2.05, 95\% CI 1.58-2.67, P <0.00001, Fig. 2b). Moreover, 6 studies with 1381 patients contributed to the analysis of the overall mortality at 1 year, and results 
Table 3 Lesion and treatment characteristics of patients

\begin{tabular}{|c|c|c|c|c|c|c|c|c|c|c|c|c|c|c|}
\hline \multirow[t]{2}{*}{ Study } & \multicolumn{2}{|c|}{ AS (\%) } & \multicolumn{2}{|c|}{ TA (\%) } & \multicolumn{2}{|c|}{ GPI (\%) } & \multicolumn{2}{|c|}{$\operatorname{IABP}(\%)$} & \multicolumn{2}{|c|}{ LAD $(\%)$} & \multicolumn{2}{|c|}{ Bifurcation (\%) } & \multicolumn{2}{|c|}{$\begin{array}{l}\text { Post TIMI } 3 \\
(\%)\end{array}$} \\
\hline & EST & LST & EST & LST & EST & LST & EST & LST & EST & LST & EST & LST & EST & LST \\
\hline Lemesle [4] & 58.9 & 60 & 43.1 & 35 & 52.9 & 41.0 & 28.0 & 10.0 & 51.0 & 42.5 & NA* & & NA* & \\
\hline Margolis [5] & NA* & & NA* & & $\mathrm{NA}^{*}$ & & $\mathrm{NA}^{*}$ & & NA* & & NA* & & NA* & \\
\hline Jones [6] & $86.5^{*}$ & & $51.5^{*}$ & & $89.0 *$ & & $\mathrm{NA}^{*}$ & & $56.2 *$ & & NA* & & NA* & \\
\hline Kimura [7] & 32.0 & 40.1 & 78.0 & 76.6 & $\mathrm{NA}^{*}$ & & 41.0 & 28.1 & 56.0 & 56.0 & 29.0 & 25.0 & 84.0 & 84.0 \\
\hline Kubo [8] & 38.7 & 53.6 & 67.2 & 72.2 & $\mathrm{NA}^{*}$ & & 25.5 & 14.4 & 45.2 & 47.5 & 62.9 & 37.4 & 88.7 & 95.0 \\
\hline Armstrong [9] & 51.2 & 66.5 & 32.1 & 33.0 & 73.9 & 67.2 & 13.4 & 9.2 & 48.0 & 38.9 & 17.3 & 14.2 & 91.6 & 94.4 \\
\hline de la TH [10] & 48.0 & 50.0 & 30.0 & 47.8 & 68.0 & 63.7 & $\mathrm{NA}^{*}$ & & 72.0 & 74.3 & 6.0 & 2.6 & 87.0 & 81.1 \\
\hline Daemen [11] & 33.0 & 48.0 & 12.0 & 12.0 & 39.0 & 30.0 & NA* & & 54.0 & 54.0 & 36.0 & 13.0 & NA* & \\
\hline Singh [12] & 15.6 & 25.0 & $\mathrm{NA}^{*}$ & & 89.2 & 88.9 & NA* & & $63.0 *$ & & $2.2 *$ & & 60.5 & 100 \\
\hline Kuramitsu [13] & $\mathrm{NA}^{*}$ & & $\mathrm{NA}^{*}$ & & $\mathrm{NA}^{*}$ & & $\mathrm{NA}^{*}$ & & 38.6 & 26.1 & 46.4 & 35.8 & NA* & \\
\hline Mahmoud [14] & $62.8 *$ & & 49.0 & 51.0 & $77.0^{*}$ & & $17.7 *$ & & $51.3 *$ & & 40.7 & & NA* & \\
\hline Lempereur [15] & 63.9 & 61.5 & 45.7 & 38.5 & $\mathrm{NA}^{*}$ & & 27.8 & 3.1 & 55.6 & 33.8 & 44.4 & 32.3 & 90.9 & 84.6 \\
\hline Kim [16] & $10.7 *$ & & $23.0^{*}$ & & $30.0 *$ & & $8.2^{*}$ & & $49.6^{*}$ & & $48.1^{*}$ & & NA* & \\
\hline Armstrong [17] & 52.7 & 68.1 & NA* & & NA* & & 13.2 & 4.0 & 40.5 & 33.4 & 6.8 & 7.3 & 85.8 & 86.4 \\
\hline Almalla 15] & $68.9 *$ & & $15.1^{*}$ & & $83.0 *$ & & NA* & & $62.3 *$ & & NA* & & NA* & \\
\hline Van Werkum [19] & $49.7 *$ & & $12.8^{*}$ & & $81.7 *$ & & NA* & & $62.4 *$ & & $51.7 *$ & & NA* & \\
\hline Katsikis [20] & 50.0 & 65.0 & $57.3 *$ & & $56.5^{*}$ & & NA* & & $43.5^{*}$ & & $3.1^{*}$ & & NA* & \\
\hline Yeo [21] & $64.0 *$ & & $58.0 *$ & & $75.0 *$ & & $16.0 *$ & & $48.0^{*}$ & & $14.0^{*}$ & & NA* & \\
\hline Konishi [22] & 25.7 & 29.3 & 66.5 & 56.1 & NA* & & NA & & 50.7 & 40.2 & 35.1 & 28.9 & NA* & \\
\hline Tovar Forero [23] & 48.0 & 70.4 & 44.3 & 50.6 & 66.6 & 47.9 & 8.5 & 6.4 & 58.6 & 48.5 & 22.6 & 18.6 & 86.1 & 91.5 \\
\hline Feldman [24] & 51.0 & 66.5 & 31.2 & 31.5 & NA* & & NA* & & NA* & & NA* & & 87.9 & 91.3 \\
\hline Shimotakahara [25] & $68.6^{*}$ & & NA* & & NA* & & NA* & & NA* & & NA* & & NA* & \\
\hline Kukreja [26] & NA* & & $\mathrm{NA}^{*}$ & & $\mathrm{NA}^{*}$ & & $\mathrm{NA}^{*}$ & & NA* & & NA* & & NA* & \\
\hline
\end{tabular}

$A S$ additional stent, $T A$ thrombus aspiration, GPI glycoprotein IIb/IIIa inhibitor, IABP intra-aortic balloon pump, ST stent thrombosis, EST early stent thrombosis, $L S T$ late stent thrombosis (including very late stent thrombosis here), LAD left anterior descending artery, TIMI thrombolysis in myocardial infarction, $N A$ not available

*Overall

showed that mortality was markedly higher in the EST group than in the LST/VLST group (RR: $1.71,95 \%$ CI 1.36-2.13, $\mathrm{P}<0.00001$, Fig. 2c). Nine studies involving 1868 patients contributed to the analysis of the overall mortality at longterm follow-up, and results demonstrated that mortality was higher in the EST group than in the LST/VLST group (RR: $1.20,95 \%$ CI 1.01-1.43, $\mathrm{P}=0.04$, Fig. 2 d).

\section{Secondary endpoints}

Regarding TVR/TLR, only one included study with 152 patients reported the incidence of TVR during hospitalization, which was similar between the EST and LST/VLST groups (EST 3.3\% vs. LST/VLST 3.28\%, P=1.00, Fig. 3a); 2 studies comprising 253 patients were used for analysis of 30-day TVR, and results showed that patients with EST had a trend toward higher risk of TVR at 30 days than patients with LST/VLST (6.3\% vs. $4.0 \%, \mathrm{P}=0.33$, Fig. $3 \mathrm{~b})$; 2 studies including 253 patients contributed to the analysis of the 1-year TVR/TLR, and results showed that the EST group had a significantly higher event rate than the LST/ VLST group (30.8\% vs. $14.2 \%, \mathrm{P}=0.002$, Fig. $3 \mathrm{c}$ ); 2 studies including 254 patients reported the incidence of TLR at long-term follow-up, which was also significantly higher in the EST group compared with the LST/VLST group $(40.1 \%$ vs. $25.8 \%, \mathrm{P}=0.009$, Fig. $3 \mathrm{~d}$ ).

In terms of RST, 3 studies with 544 patients, 3 studies with 556 patients, 2 studies with 404 patients, and 3 studies with 766 patients contributed to the analysis of the overall incidence of RST during hospitalization, at 30 days, at 1 year and at long-term follow-up, respectively. The results showed that patients with EST had a trend toward higher risk of RST during hospitalization (3.8\% vs. $2.4 \%, \mathrm{P}=0.39$, Fig. $4 \mathrm{a})$, at 30 days $(7.2 \%$ vs. $3.4 \%, P=0.33$, Fig. $4 b)$, at 1 year $(9.1 \%$ vs. $5.7 \%, \mathrm{P}=0.20$, Fig. $4 \mathrm{c})$ and at long-term follow-up $(7.6 \%$ vs. $4.7 \%, \mathrm{P}=0.05$, Fig. $4 \mathrm{~d})$, although differences were not statistically significant. 


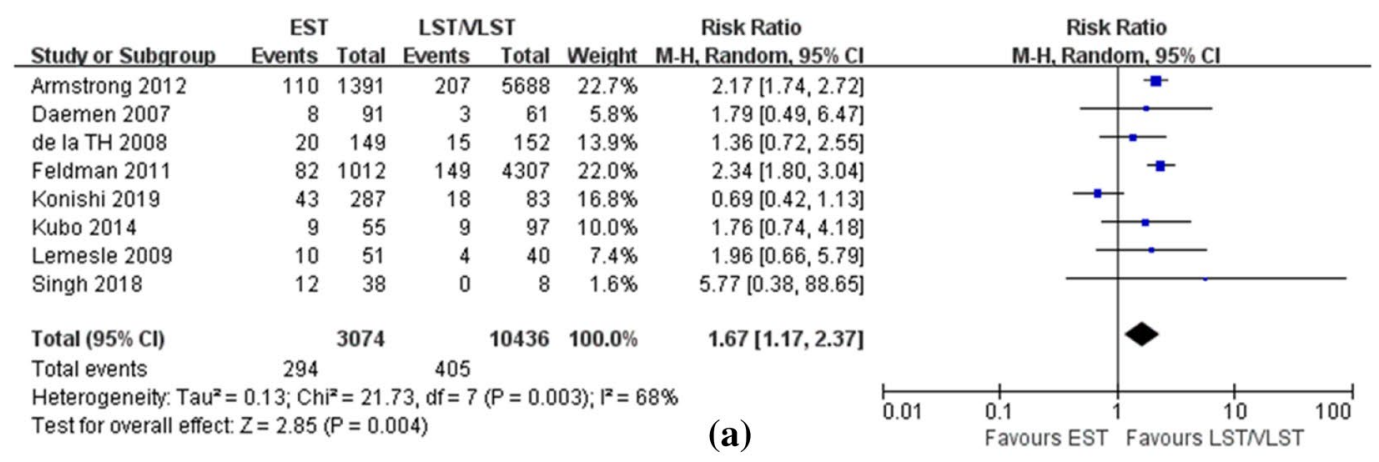

\begin{tabular}{|c|c|c|c|c|c|c|c|c|c|c|}
\hline Study or Subgroup & $\begin{array}{l}\text { EST } \\
\text { Events }\end{array}$ & Total & $\begin{array}{l}\text { LSTNL } \\
\text { Events }\end{array}$ & $\begin{array}{l}\text { ST } \\
\text { Total }\end{array}$ & Weight & $\begin{array}{c}\text { Risk Ratio } \\
\text { M-H, Fixed, } 95 \% \mathrm{Cl}\end{array}$ & \multicolumn{4}{|c|}{$\begin{array}{c}\text { Risk Ratio } \\
\text { M-H, Fixed, } 95 \% \mathrm{Cl}\end{array}$} \\
\hline Armstrong 2014 & 17 & 129 & 20 & 527 & $11.2 \%$ & $3.47[1.87,6.44]$ & & & $\rightarrow$ & \\
\hline Daemen 2007 & 9 & 91 & 4 & 61 & $6.8 \%$ & $1.51[0.49,4.68]$ & & & & \\
\hline Kimura 2010 & 48 & 322 & 28 & 289 & $42.0 \%$ & $1.54[0.99,2.38]$ & & & $=$ & \\
\hline Kuramitsu 2019 & 33 & 179 & 14 & 134 & $22.8 \%$ & $1.76[0.98,3.16]$ & & & $\rightarrow-$ & \\
\hline Lemesle 2009 & 10 & 51 & 5 & 40 & $8.0 \%$ & $1.57[0.58,4.22]$ & & & & \\
\hline Lempereur 2016 & 8 & 36 & 4 & 65 & $4.1 \%$ & $3.61[1.17,11.17]$ & & & & \\
\hline Mahmoud 2011 & 12 & 59 & 3 & 54 & $4.5 \%$ & $3.66[1.09,12.28]$ & & & & \\
\hline Margolis 2016 & 4 & 35 & 0 & 48 & $0.6 \%$ & $12.25[0.68,220.39]$ & & & & \\
\hline Total $(95 \% \mathrm{Cl})$ & & 902 & & 1218 & $100.0 \%$ & $2.05[1.58,2.67]$ & & & 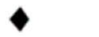 & \\
\hline Total events & 141 & & 78 & & & & & & & \\
\hline $\begin{array}{l}\text { Heterogeneity: } \mathrm{Chi}^{2}= \\
\text { Test for overall effect }\end{array}$ & $\begin{array}{l}8.58, d f= \\
Z=5.37\end{array}$ & $\begin{array}{l}7(P= \\
P<0.0\end{array}$ & $\begin{array}{l}0.28) ;\left.\right|^{2}= \\
00001)\end{array}$ & $=18 \%$ & & (b) & 0.005 & $\begin{array}{c}0.1 \\
\text { Favours EST }\end{array}$ & $\begin{array}{r}10 \\
\text { Favours L }\end{array}$ & $\begin{array}{l}0 \\
\text { LSTMLST }^{200}\end{array}$ \\
\hline
\end{tabular}

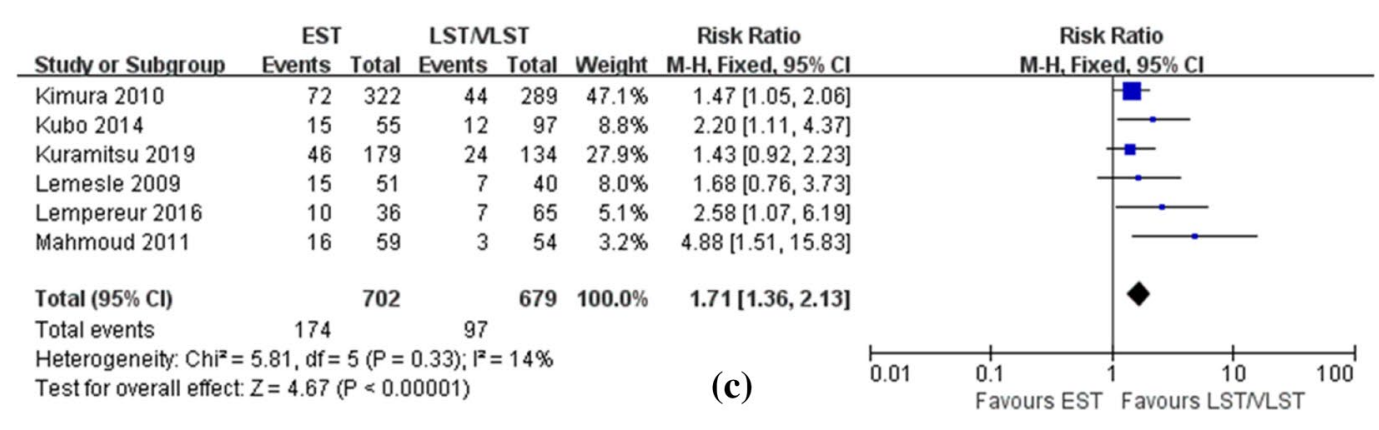

$\begin{array}{llll}\text { EST } & \text { RSTMLS Ratio } & \text { Risk Ratio }\end{array}$

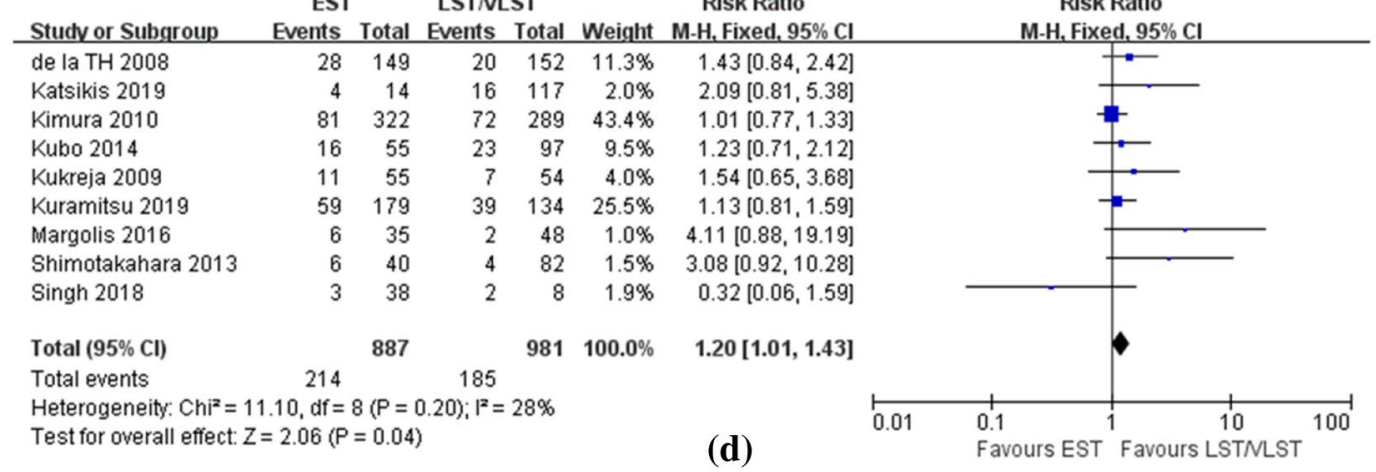

Fig. 2 Forest plot with RR for EST vs LST/VLST (a) in-hospital mortality (b) 30-day mortality (c) 1-year mortality (d) long-term mortality 


\section{Other outcomes of interest}

One study including 152 patients reported the incidence of cardiac death (CD) at 1 year and at long-term follow-up, which were both numerically higher in the EST group than in the LST/VLST group (1-year: $23.6 \%$ vs. $11.3 \%, \mathrm{P}=0.05$; long-term: $25.5 \%$ vs. $18.6 \%, \mathrm{P}=0.31)$. One study including 91 patients reported the rates of myocardial infarction (MI) during hospitalization, at 30 days and at 1 year, which were both numerically higher in the EST group than in the LST/VLST group (in-hospital: $27.5 \%$ vs. $20.0 \%$, $\mathrm{P}=0.42$; 30 -day: $31.4 \%$ vs. $25.0 \%, \mathrm{P}=0.31 ; 1$ year: $37.2 \%$ vs. $35.0 \%, \mathrm{P}=0.82)$. Eight studies reported the incidence of major adverse cardiovascular events (MACE, defined as the combined endpoints of various outcomes), which were also higher in the EST group than in the LST/VLST group (Supplemental Table 2).

\section{Discussion}

To the best of our knowledge, this is the first meta-analysis to investigate the associations between the timing of ST occurrence and the clinical outcomes of ST. Results showed that patients with EST had worse clinical outcomes than patients with LST/VLST in both short- and long-term follow-up after PCI treatment.

The poor clinical outcomes in EST patients were consistent with the poor angiographic outcomes in this post-PCI entity. In the present study, the rate of achievement of postPCI TIMI flow grade 3 was significantly lower in the EST group than in the LST/VLST group. Additionally, several studies that performed quantitative coronary angiographic analysis found that patients with EST had a smaller minimum luminal diameter and a higher percentage of diameter

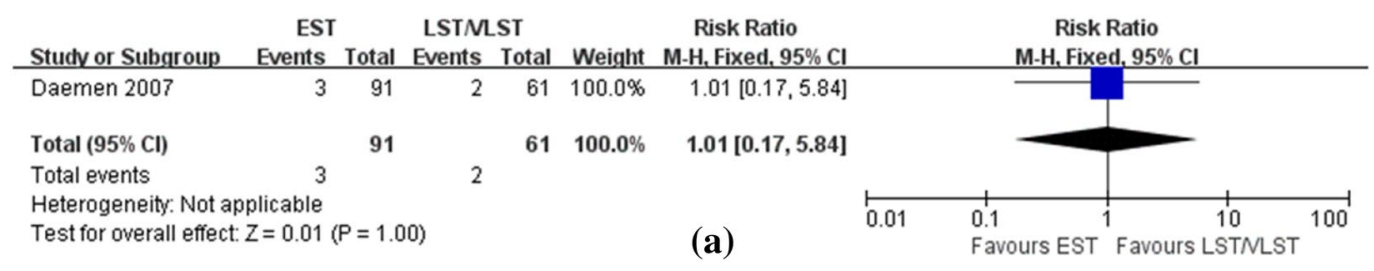

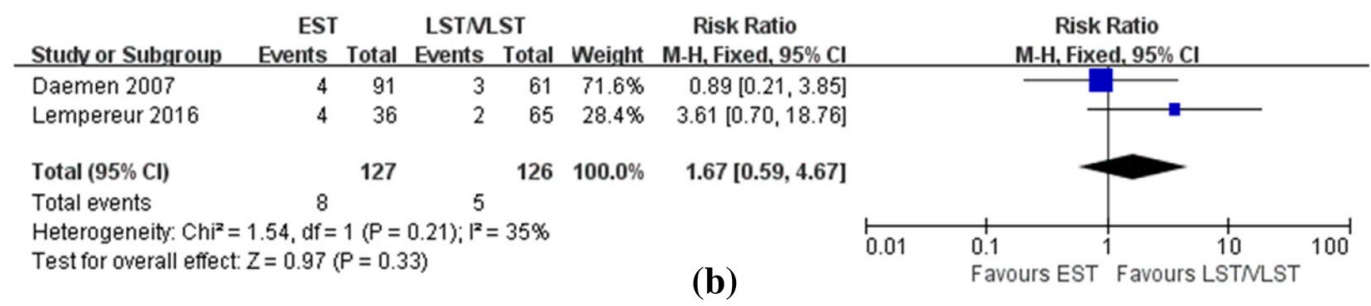

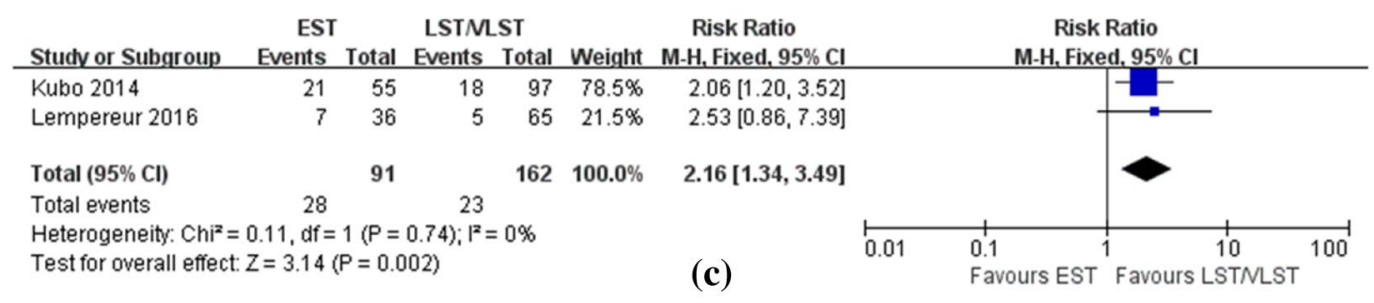

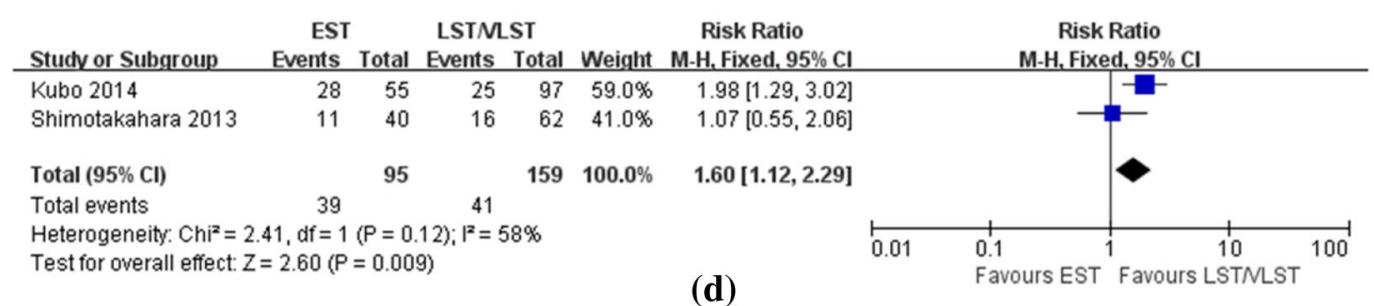

Fig. 3 Forest plot with RR for EST vs LST/VLST (a) in-hospital TVR (b) 30-day TVR (c) 1-year TVR/TLR (d) long-term TLR 


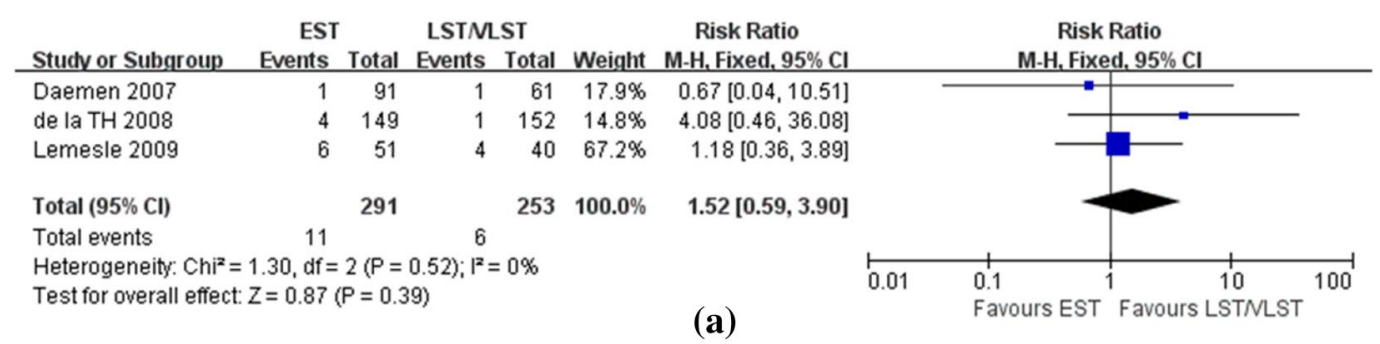

(a)

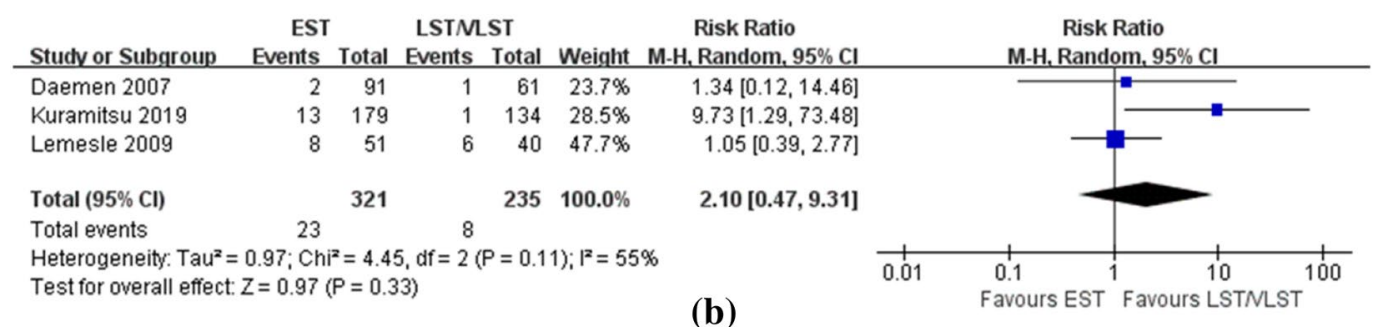

\begin{tabular}{|c|c|c|c|c|c|c|c|c|c|c|}
\hline Study or Subgroup & $\begin{array}{r}\text { EST } \\
\text { Events }\end{array}$ & Total & $\begin{array}{l}\text { LSTML } \\
\text { Events }\end{array}$ & $\begin{array}{l}\text { ST } \\
\text { Total }\end{array}$ & Weight & $\begin{array}{c}\text { Risk Ratio } \\
\text { M-H, Fixed, 95\% Cl }\end{array}$ & & $\begin{array}{r}\text { Risk } \\
\text { M-H, Fixe }\end{array}$ & $\begin{array}{l}\text { Ratio } \\
\text { d. } 95 \% \mathrm{Cl}\end{array}$ & \\
\hline Kuramitsu 2019 & 13 & 179 & 4 & 134 & $40.5 \%$ & $2.43[0.81,7.29]$ & & & $=$ & \\
\hline Lemesle 2009 & 8 & 51 & 6 & 40 & $59.5 \%$ & $1.05[0.39,2.77]$ & & & & \\
\hline Total $(95 \% \mathrm{Cl})$ & & 230 & & 174 & $100.0 \%$ & $1.61[0.78,3.30]$ & & & & \\
\hline Total events & 21 & & 10 & & & & & & & \\
\hline $\begin{array}{l}\text { Heterogeneity: } \mathrm{Chi}^{2} \\
\text { Test for overall effec }\end{array}$ & $\begin{array}{l}1.30, d f= \\
z=1.29\end{array}$ & $\begin{array}{l}1(P= \\
P=0.2\end{array}$ & $\begin{array}{l}0.26) ; 1^{2}= \\
0)\end{array}$ & $=23 \%$ & & & 0.01 & $\begin{array}{l}0.1 \\
\text { Favours EST }\end{array}$ & 1 Favours L & $\begin{array}{l}10 \\
10 \\
\text { LSTMLST }\end{array}$ \\
\hline
\end{tabular}

(c)

\begin{tabular}{|c|c|c|c|c|c|c|c|c|c|c|}
\hline Studv or Subgroup & $\begin{array}{r}\text { EST } \\
\text { Events }\end{array}$ & Total & $\begin{array}{l}\text { LSTML } \\
\text { Events }\end{array}$ & $\begin{array}{l}\text { ST } \\
\text { Total }\end{array}$ & Weight & $\begin{array}{c}\text { Risk Ratio } \\
\text { M-H, Fixed, } 95 \% \mathrm{Cl}\end{array}$ & & $\begin{array}{r}\text { Risk I } \\
\text { M-H, Fixe }\end{array}$ & $\begin{array}{l}\text { Ratio } \\
\text { d. } 95 \% \mathrm{CI}\end{array}$ & \\
\hline de la TH 2008 & 9 & 149 & 5 & 152 & $30.9 \%$ & $1.84[0.63,5.35]$ & & & & \\
\hline Kubo 2014 & 7 & 55 & 9 & 97 & $40.6 \%$ & $1.37[0.54,3.48]$ & & & $=$ & \\
\hline Kuramitsu 2019 & 13 & 179 & 4 & 134 & $28.5 \%$ & $2.43[0.81,7.29]$ & & & & \\
\hline Total $(95 \% \mathrm{Cl})$ & & 383 & & 383 & $100.0 \%$ & $1.82[1.01,3.28]$ & & & & \\
\hline Total events & 29 & & 18 & & & & & & & \\
\hline $\begin{array}{l}\text { Heterogeneity: Chiz } \\
\text { Test for overall effec }\end{array}$ & $\begin{array}{l}0.62, d f= \\
Z=1.98\end{array}$ & $\begin{array}{l}2(P=0 \\
P=0.0\end{array}$ & $\begin{array}{l}0.73): 1^{2}= \\
\text { 5) }\end{array}$ & $=0 \%$ & & (d) & 0.01 & $\begin{array}{l}0.1 \\
\text { Favours EST }\end{array}$ & Favours & $\begin{array}{l}10 \\
10 \\
\text { LSTMLST }\end{array}$ \\
\hline
\end{tabular}

Fig. 4 Forest plot with RR for EST vs LST/VLST (a) in-hospital RST (b) 30-day RST (c) 1-year RST (d) long-term RST

stenosis at the end of procedure as well as at long-term angiographic follow-up than those with LST/VLST $[4,8,11,13]$.

These findings can possibly be explained as follows. First, previous studies have demonstrated that patients who develop EST are usually those with adverse baseline characteristics such as DM, STEMI, CS and multivessel diseases $[1,28]$. Similarly, the present study found that patients with EST had a higher rate of DM, bifurcation lesions and LAD lesions than those with LST/VLST. This high baseline risk profile in EST patients may explain per se the poor efficacy of PCI and the higher rate of unfavorable outcomes in this entity [18, 19, 21, 23]. Moreover, the clinical presentation at time of ST was also more disastrous in EST patients than it was in LST/VLST patients. As observed in the present study, the rates of CS and IABP use at the time of presentation were higher in the EST group than in the LST/VLST group. This finding could partly explain the higher mortality in EST patients, because CS has been shown to be associated with in-hospital mortality as high as $48 \%$ and 1-year mortality as high as $58 \%$ despite aggressive treatment therapies [29].

Second, the present study also found that in patients with EST, surgeons tended to restore vessel patency by balloon angioplasty only, whereas in patients with LST/VLST, they preferred to utilize a new stent. This finding was in line with the assumption that more stent deployment-related issues may be noted in EST patients. Previous studies using intravascular imaging have identified stent underexpansion and acute malapposition (occurring during index procedure) 
as the most prevalent abnormalities in patients with EST. Whereas, late malapposition (occurring during follow-up), delayed endothelialization (manifesting as uncovered struts) and neoatherosclerosis have been regarded as the most important mechanisms for LST/VLST [30-33]. Moreover, the higher rates of utilizing GPI among patients with EST suggests that a higher thrombus burden may be present in this critically ill subgroup $[34,35]$. Therefore, patients with EST were more likely to face more difficult and complex challenges during PCI, which may further lead to poor outcomes.

Finally, the higher adverse events rate in patients with EST may be related in part to damaged coronary collaterals. Indeed, collaterals can minimize injury to the myocardium at the time of the event and result in better outcomes $[36,37]$. In patients with LST/VLST, the thrombus formation was more like a progressive evolution, thus, there was enough time for collateral circulation to develop. However, in patients with EST, the ability of establishing coronary collateral circulation may be impaired by the rapid onset of stent thrombosis due to the higher on-treatment platelet reactivity [38], which may lead to a larger myocardium infarct size and higher rates of adverse events.

Treatment of EST appears to be more challenging than that of LST/VLST, and no specific guidelines exist for optimal strategies for addressing EST. A two-step approach may be more suitable for EST. The study of Carrick et al. [39] demonstrated that, in high-risk STEMI patients, deferred stenting is associated with fewer intraprocedural thrombotic events, higher TIMI flow grade and increased myocardial salvage compared with immediate stenting. Similarly, a recent meta-analysis including 744 patients demonstrated that a deferred stent implantation strategy was associated with improved TIMI flow grade, greater TIMI myocardial blush grade and decreased MACEs without increasing major bleeding events in STEMI patients with a high thrombus burden [40]. Besides, it has been suggested that use of intravascular imaging including intravascular ultrasound (IVUS) and optical coherence tomography (OCT), which ascertains the predisposing mechanical factors of ST, may be a potential adjunctive therapy for EST [41, 42].Thus, it seems reasonable to consider that aggressive EST cases can benefit from a deferred PCI strategy with intra-coronary imaging after optimal medical therapy. Further studies are required to evaluate this speculative approach.

In the present study, heterogeneity was either low or moderate in the results of primary and secondary endpoints, but a high degree of heterogeneity was noted in the analysis of inhospital mortality $\left(\mathrm{I}^{2}=68 \%\right)$. Differences in patients' clinical manifestations between the study of Konishi et al. [22] and others may account for this heterogeneity. Patients presenting with AMI at the time of ST only accounted for $29.5 \%$ in the Konishi study, whereas it accounted for 70\%-90\% in the other studies (Table 1). When removing the Konishi study, the heterogeneity disappeared $\left(\mathrm{I}^{2}=0 \%\right)$ and the results became more significant $(\mathrm{P}<0.00001$, Supplemental Fig. 8).

A discrepancy regarding CKD incidence was found between studies in the EST and LST/VLST group. A possible explanation is that a significant difference exists in the incidence of CKD between patients with VLST and patients with LST (the rate was lower in VLST than in LST) [7-9, $13,15,17]$, which may lead to a certain level of bias when calculating the overall rate of CKD for the combined LST/ VLST group.

Finally, male gender and dyslipidemia were found to be more frequent in patients with LST/VLST, but no dramatic evidence was found of the association between gender or dyslipidemia and the prognosis of ST, except for one study that found male gender was associated with MI at long-term follow-up [23], and another study that identified dyslipidemia as an independent predictor of composite CD and MI at five years after PCI [8].

\section{Limitations}

First, this meta-analysis shared the limitations of the original studies. Second, the results of TVR/TLR and RST needed to be interpreted with care since the analysis might be too small to properly detect statistical differences between the two groups. Third, definitions of MACE in the individual studies were significantly different and there were limited studies reporting the rate of $\mathrm{MI}$ and $\mathrm{CD}$, we were therefore unable to conduct subgroup analyses of these outcomes of interest in the present study. Fourth, with regard to the methods, it would have been more appropriate to include a negative control group (patients without ST after PCI) and compare it with results of the EST group and the LST/ VLST group. However, only one study was enrolled that established a negative control group [13], therefore, such overall comparison was not possible. Fifth, we included studies using various types of stents (BMS and DES, firstgeneration DES and second-generation DES, polymer stent and polymer-free stents, etc.) during the index procedure, but meaningful subgroup analysis according to the initial types of stents could not be performed due to the insufficient data of the original articles. Finally, limited data regarding the strategy of antiplatelet use after ST also hampered our ability to explore the effects of this important measure for outcomes of EST and LST/VLST.

\section{Conclusions}

After PCI treatment, patients with EST have worse clinical outcomes in both short- and long-term follow-up than patients with LST/VLST. Treatment for EST patients 
remains challenging, and further studies are needed that concentrate on determining the optimal treatment strategies for EST.

Funding This work was supported, in part, by National 135 Key Research and Development Program in 2016 (No.2016YFC1301203); Major Science and Technology Projects of Tianjin Science and Technology Commission in 2016 (No. 16ZXMJSY00150); The Key Project of Healthcare Industry of Tianjin in 2016 (No.16KG131); The Science and Technology Project of Tianjin Jin nan District Science and Technology Commission (No.20171514).

\section{Compliance with ethical standards}

Conflict of interest The authors declare that they have no conflict of interest.

Open Access This article is licensed under a Creative Commons Attribution 4.0 International License, which permits use, sharing, adaptation, distribution and reproduction in any medium or format, as long as you give appropriate credit to the original author(s) and the source, provide a link to the Creative Commons licence, and indicate if changes were made. The images or other third party material in this article are included in the article's Creative Commons licence, unless indicated otherwise in a credit line to the material. If material is not included in the article's Creative Commons licence and your intended use is not permitted by statutory regulation or exceeds the permitted use, you will need to obtain permission directly from the copyright holder. To view a copy of this licence, visit http://creativecommons.org/licenses/by/4.0/.

\section{References}

1. Iqbal J, Sumaya W, Tatman V et al (2013) Incidence and predictors of stent thrombosis: a single-centre study of 5,833 consecutive patients undergoing coronary artery stenting. EuroIntervention 9:62-69

2. Rozemeijer R, Wing WC, Leenders G et al (2019) Incidence, angiographic and clinical predictors, and impact of stent thrombosis: a 6-year survey of 6,545 consecutive patients. Neth Heart J 27:321-329

3. Cutlip DE, Windecker S, Mehran R et al (2007) Clinical end points in coronary stent trials: a case for standardized definitions. Circulation 115:2344-2351

4. Lemesle G, De Labriolle A, Bonello L et al (2009) Clinical manifestation and prognosis of early versus late stent thrombosis of drug-eluting stents. J Interv Cardiol 22:228-233

5. Margolis G, Barkagan M, Flint N et al (2016) Prevalence and outcomes of early versus late stent thrombosis presenting as ST-segment elevation myocardial infarction. Coron Artery Dis 27:667-672

6. Jones DA, Gallagher S, Rathod KS et al (2013) Contemporary analysis of incidence and outcomes of stent thrombosis presenting as ST elevation myocardial infarction in a primary percutaneous coronary intervention cohort. Am J Cardiol 112:1347-1354

7. Kimura T, Morimoto T, Kozuma K et al (2010) Comparisons of baseline demographics, clinical presentation, and long-term outcome among patients with early, late, and very late stent thrombosis of sirolimus-eluting stents: Observations from the Registry of Stent Thrombosis for Review and Reevaluation (RESTART). Circulation 122:52-61
8. Kubo S, Kadota K, Ichinohe T et al (2014) Comparison of longterm outcome after percutaneous coronary intervention for stent thrombosis between early, late, and very late stent thrombosis. Circ J 78:101-109

9. Armstrong EJ, Feldman DN, Wang TY et al (2012) Clinical presentation, management, and outcomes of angiographically documented early, late, and very late stent thrombosis. JACC Cardiovasc Interv 5:131-140

10. de la Torre-Hernández JM, Alfonso F, Hernández F et al (2008) Drug-eluting stent thrombosis: results from the multicenter Spanish registry ESTROFA (Estudio ESpañol sobre TROmbosis de stents FArmacoactivos). J Am Coll Cardiol 51:986-990

11. Daemen J, Wenaweser P, Tsuchida K et al (2007) Early and late coronary stent thrombosis of sirolimus-eluting and paclitaxeleluting stents in routine clinical practice: data from a large twoinstitutional cohort study. Lancet 369:667-678

12. Singh B, Ramesh B, Rajendran $R$ et al (2018) Incidence, predictors, and long term clinical outcome of angiographic definite stent thrombosis in real world scenario - A prospective cohort study. Cardiovasc Revasc Med 19:666-670

13. Kuramitsu S, Ohya M, Shinozaki T et al (2019) Risk factors and long-term clinical outcomes of second-generation drug-eluting stent thrombosis. Circ Cardiovasc Interv 12:e007822

14. Mahmoud KD, Vlaar PJ, van den Heuvel AF et al (2011) Usefulness of thrombus aspiration for the treatment of coronary stent thrombosis. Am J Cardiol 108:1721-1727

15. Lempereur M, Bogale N, Fahmy P et al (2016) Clinical characteristics, angiographic findings, and one-year outcome of 101 consecutive stent thrombosis cases in British Columbia. Cardiovasc Revasc Med 17:74-80

16. Kim MC, Kim IS, Jeong MH et al (2019) Incidence of cardiac death and recurrent stent thrombosis after treatment for angiographically confirmed stent thrombosis. J Cardiol 74:267-272

17. Armstrong EJ, Maddox TM, Carey EP et al (2014) Mortality after presentation with stent thrombosis is associated with time from index percutaneous coronary intervention: a report from the VA CART program. Am Heart J 168:560-567

18. Almalla M, Schröder J, Hennings V et al (2013) Long-term outcome after angiographically proven coronary stent thrombosis. Am J Cardiol 11:1289-1294

19. van Werkum JW, Heestermans AA, de Korte FI et al (2009) Longterm clinical outcome after a first angiographically confirmed coronary stent thrombosis: an analysis of 431 cases. Circulation 119:828-834

20. Katsikis A, Keeble TR, Davies JR et al (2019) Contemporary management of stent thrombosis: Predictors of mortality and the role of new-generation drug-eluting stents. Catheter Cardiovasc Interv. https://doi.org/10.1002/ccd.28467

21. Yeo KK, Armstrong EJ, Soni K et al (2015) Long-term outcomes of angiographically confirmed coronary stent thrombosis: results from a multicentre California registry. EuroIntervention 11:188-195

22. Konishi A, Mitsutake Y, Ho M et al (2019) Patient and lesion characteristics in late/very late stent thrombosis with everolimuseluting stents from real-world adverse event reporting. J Cardiol. https://doi.org/10.1016/j.jjcc.2019.07.016

23. Tovar FMN, Zanchin T, Masdjedi K et al (2019) Incidence and predictors of outcomes after a first definite coronary stent thrombosis. EuroIntervention. https://doi.org/10.4244/EIJ-D-19-00219

24. Feldman DN, Armstrong EJ, Wang TY et al (2011) Presentation treatment and outcomes of early late and very late stent thrombosis: a report from the american college of cardiology National Cardiovascular Data Registry. J Am Coll Cardiol 57:E1103

25. Shimotakahara J, Yamaji K, Iwabuchi M et al (2013) Long-term outcomes in patients with early, late and very late stent thrombosis after bare metal stenting. Eur Heart J 34:4897-4897 
26. Kukreja N, Onuma Y, Garcia-Garcia HM et al (2009) The Risk of Stent Thrombosis in Patients With Acute Coronary Syndromes Treated With Bare-Metal and Drug-Eluting Stents. JACC Cardiovasc Interv 2:534-541

27. The Newcastle-Ottawa Scale (NOS) for assessing the quality of nonrandomised studies in meta-analyses (2016) https://www.ohri. ca/programs/clinical_epidemiology/oxford.asp. Accessed 4 Mar 2016.

28. van Werkum JW, Heestermans AA, Zomer AC et al (2009) Predictors of Coronary Stent Thrombosis: The Dutch Stent Thrombosis Registry. J Am Coll Cardiol 53:1399-1409

29. Aissaoui N, Puymirat E, Delmas C et al (2020) Trends in cardiogenic shock complicating acute myocardial infarction. EUR J HEART FAIL 22:664-672

30. Ong DS, Jang IK (2015) Causes, assessment, and treatment of stent thrombosis-intravascular imaging insights. Nat Rev Cardiol $12: 325-336$

31. Adriaenssens T, Joner M, Godschalk TC et al (2017) Optical Coherence Tomography Findings in Patients With Coronary Stent Thrombosis: A Report of the PRESTIGE Consortium (Prevention of Late Stent Thrombosis by an Interdisciplinary Global European Effort). Circulation 136:1007-1021

32. Parodi G, La Manna A, Di VL et al (2013) Stent-related defects in patients presenting with stent thrombosis: differences at optical coherence tomography between subacute and late/very late thrombosis in the Mechanism of Stent Thrombosis (MOST) study. EuroIntervention 9:936-944

33. Souteyrand G, Amabile N, Mangin L et al (2016) Mechanisms of stent thrombosis analysed by optical coherence tomography: insights from the national PESTO French registry. Eur Heart J 37:1208-1216

34. Chechi T, Vecchio S, Vittori G et al (2008) ST-segment elevation myocardial infarction due to early and late stent thrombosis. A new group of high-risk patients. J Am Coll Cardiol 51:2396-2402

35. Sianos G, Papafaklis MI, Daemen J et al (2007) Angiographic stent thrombosis after routine use of drug-eluting stents in
ST-segment elevation myocardial infarction: the importance of thrombus burden. J Am Coll Cardiol 50:573-583

36. Cui K, Lyu S, Song X et al (2018) Effect of coronary collaterals on prognosis in patients undergoing primary percutaneous coronary intervention for acute ST-segment elevation myocardial infarction: a meta-analysis. Angiology 69:803-811

37. Freund A, Stiermaier T, de Waha-Thiele S et al (2020) Coronary collaterals in patients with ST-elevation myocardial infarction presenting late after symptom onset. Clin Res Cardiol. https:// doi.org/10.1007/s00392-020-01625-w

38. Geisler T, Zurn C, Simonenko R et al (2010) Early but not late stent thrombosis is influenced by residual platelet aggregation in patients undergoing coronary interventions. Eur Heart J 31:59-66

39. Carrick D, Oldroyd KG, McEntegart M et al (2014) A randomized trial of deferred stenting versus immediate stenting to prevent noor slow-reflow in acute ST-segment elevation myocardial infarction (DEFER-STEMI). J Am Coll Cardiol 63:2088-2098

40. Sun B, Liu J, Yin H et al (2019) Delayed vs. immediate stenting in STEMI with a high thrombus burden: a systematic review and meta-analysis. Herz 44:726-734

41. Mahtta D, Mahmoud AN, Mojadidi MK et al (2018) Intravascular ultrasound-guided percutaneous coronary intervention: an updated review. Cardiovasc Innov Appl. https://doi.org/10.15212 /CVIA.2017.0029

42. Kubo T, Imanishi T, Takarada S et al (2007) Assessment of culprit lesion morphology in acute myocardial infarction: ability of optical coherence tomography compared with intravascular ultrasound and coronary angioscopy. J Am Coll Cardiol 50:933-939

Publisher's Note Springer Nature remains neutral with regard to jurisdictional claims in published maps and institutional affiliations. 